\title{
ON THE DETECTION AND LOCALIZATION OF SHIN COLLISIONS AND REACTIVE ACTIONS IN QUADRUPED ROBOTS
}

\author{
VICTOR BARASUOL, GEOFF FINK, MICHELE FOCCHI, \\ DARWIN G. CALDWELL and CLAUDIO SEMINI \\ Dynamic Legged Systems, Istituto Italiano di Tecnologia (IIT), Morego st., 30, 16163, Genoa, Italy \\ E-mail: <first name>.<last name>@iit.it
}

\begin{abstract}
In this work, we investigate the impact of shin (distal limb) collisions on the performance of quadruped locomotion. In particular, we carry out a detailed study on the locomotion performance sensitivity to systematic errors and delays in the estimation of the shin contact location. We propose a sensor-less model based on kinematics to estimate the location of a single contact point at the shin level. In order to improve the robustness of robot locomotion, we use insights from our sensitivity study and our proposed model to develop a reactive strategy to detect and feedback information about the contact into the trunk controller. The effectiveness of the proposed approaches is experimentally demonstrated on the HyQ robot.
\end{abstract}

Keywords: Shin collision, contact detection, contact localization, legged locomotion, quadruped robots

\section{Introduction}

Unexpected collisions are very likely to occur in legged robots when traversing unstructured terrain. However, many research works assume perfect knowledge of the environment and focus on generating motions at locations that avoid collisions. On the other hand, information about the topology of the environment might not be available in real-world applications. In these cases, the robot-environment interaction is not guaranteed to happen only at the end-effector, i.e., the hands or feet of the robot. This situation is challenging because most advanced control strategies used to stabilize the trunk require the exact location and Jacobian of the contact points. ${ }^{1-3}$ Moreover, the inclination of the contact surface and its frictional properties are also necessary to properly distribute the contact forces.

Consequently, to achieve robust locomotion, it is important to detect unexpected collisions and use them as feedback for the stabilizing trunk controller. Relevant research effort has been devoted to finding methods that detect and estimate the location of arbitrary contacts occurring anywhere along the robot structure (not only at feet and hands).

Some approaches use force/torque $(\mathrm{F} / \mathrm{T})$ sensors combined with a distributed skin to capture external forces that are applied to arbitrary locations. ${ }^{4-7}$ However, most robots do not have this type of sensor as they significantly increase the complexity of the platform. Furthermore, most of these sensors are not capable nor designed to handle strong interaction forces; they are typically designed for tasks involving human-robot interaction.

Other methods for detecting and estimating external forces are based on model-based observers of generalized momenta $(\mathrm{GM}){ }^{8}$ The basic idea of these approaches is to compare the computed torque with the real torque to infer the action of the external forces. Their advantage is that they require only proprioceptive measurements (torque and encoder measurement) without the need of additional force sensors.

De Luca et al. ${ }^{9}$ presented the first approach able to simultaneously detect collisions, find the contact link (not the contact location) and estimate the external forces. Contact 
isolation $^{\mathrm{a}}$ in most GM approaches rely on thresholding the estimation coming from an observer (termed residual) that is a filtered version of the external wrench. However, most (GM approaches are applied only to fixed-base robots. Vorndamme et al. developed an approach for floating-base robots (humanoids) in which they combined and compared the GM approach with a F/T-sensor-based approach. ${ }^{10}$ In addition to contact isolation, some approaches try to estimate the contact location at the price of increased complexity, e.g., Manuelli et al. proposed an optimization-based approach based on particle filters to detect and localize multiple contacts on a humanoid using only proprioceptive sensors. ${ }^{11}$ In general, the majority of approaches based on proprioceptive measurements fail to determine the exact contact location on the most distal link. Also both Vorndamme et al. ${ }^{10}$ and Manuelli et al. ${ }^{11}$ rely on a very accurate model of the robot. Moreover, they are demonstrated only in simulation with a robot that is stationary when an external force is applied. They do not consider the implications of what happens when the robot contact is made during motion.

When it comes to real applications, all the approaches based on filters or observers are strongly affected by modeling errors, sensor noise, offsets, friction and structural compliance. Commonly, these aspects present themselves as an increase in estimation errors. Moreover, due to the fact that they are filter-based, these approaches present an inherent delay (e.g., due to filter dynamics or the time it takes to reach the threshold to trigger the detection) that often poses a limit on the gains of the observers.

In the context of quadruped robots, shin collisions are typically undesired events. Depending on the configurations of the robot leg, i.e., knee bent forward (KBF) or knee bent backward (KBB), ${ }^{12}$ shin collisions can cause the robot to get stuck when moving forward or backward. During blind locomotion this situation becomes even more critical since the robot cannot acquire detailed information about the surface below it.

In this work, we investigate the effect that systematic errors and delays in contact estimation have on quadruped locomotion performance. Initially, we describe a study of the impact of shin collisions on dynamic locomotion, then we propose a kinematic strategy to localize collisions on the most distal joint (i.e., the shin). We believe the approach is generic enough to be extended to humanoids. We demonstrate with real experiments the increase in locomotion performance given by exploiting the knowledge of the (online) estimated shin contact in the stabilizing controller. To the best of the author's knowledge this is the first time that a strategy based on shin collision detection has been successfully applied to a real quadruped robot.

The contributions of this paper can be summarized as follows:

- A study about the effect of shin collisions during quadruped locomotion and a proposal on how to react to them independent of whether the shin-obstacle contact position can or cannot be estimated;

- The impact of systematic errors and delays in the estimation of the shin collision location;

- A simple model for a sensor-less strategy to detect shin collisions and estimate the shin-obstacle contact position;

- Hardware experiments closing the loop with an optimization-based trunk controller ${ }^{2}$ where the output of the shin estimator is exploited in the optimization.

\section{Locomotion sensitivity to the shin collisions and their detection}

This section contains one of the main contributions presented in this paper: the study of the locomotion sensitivity to shin collisions. The conclusions of this study are the basis

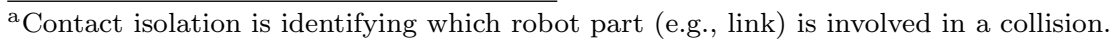


of the reactive strategies proposed in the following sections. For this study, we considered a walking trot because it is currently the most commonly chosen dynamic gait for rough terrain locomotion and it is very sensitive to shin collisions.

Our study aims to understand how delayed detections and systematic errors in the contact localization affect the locomotion performance. To answer these questions, we created an ideal simulation scenario in Gazebo where we required $\mathrm{HyQ}^{13}$ to step over a $16 \mathrm{~cm}$ high platform while trotting at a certain speed as seen in Fig. 1 (left). Virtual shin sensors (one for each leg) are able to detect a collision at the shin and localize the position on the shin where the collision occurs.

In this simulation, the motion generation and the control is done by the Reactive Controller Framework $^{14}$ (RCF). The RCF has a trunk stabilization module that uses leg contact Jacobians, that, by default considers all contacts happening at the foot level. ${ }^{2}$ Our trunk controller also uses the information about the surface inclination and the friction coefficient to generate ground reaction forces that respect the associated friction cone. We call the reactive action to a shin collision the stance reflex. When a shin collision occurs our proposed stance reflex uses the information coming from the virtual shin sensor to update the contact Jacobian, the friction coefficient and the orientation of the friction cone. It is important to note that when a shin collision happens, the friction cone, which was previously defined by the surface inclination, becomes defined by the orientation of the shin as illustrated in Fig. 1 (left) by the translucent red cones.

To understand the impact of delayed detections and systematic localization errors (i.e., constant offset error) on the locomotion performance, we ran hundreds of simulations considering the following parameter variations: two shin-obstacle friction coefficients $(0.6$ and $0.9)$; two robot forward velocities $(0.2 \mathrm{~m} / \mathrm{s}$ and $0.35 \mathrm{~m} / \mathrm{s})$, a range of detection delays $(0 \mathrm{~ms}$ to $160 \mathrm{~ms})$; and a range of systematic localization errors $(-8 \mathrm{~cm}$ to $8 \mathrm{~cm})$. For statistical reasons, we ran five trials for every possible combination. Figure 1 (right) shows the results. In the figure each 5-trial set is represented by a red cross (unsuccessful) or by a large cyan dot (successful). An unsuccessful set is when the robot has failed at least once, when trying to step on the platform with the hind legs. In this case, the robot gets stuck because the hind shins keep slipping on the platform edge. As locomotion parameters, the robot trots with a step frequency of $1.3 \mathrm{~Hz}$ and a duty factor of $0.65^{\mathrm{b}}$. Given that these values result in a stance period of $0.5 \mathrm{~s}$, the maximum detection delay of $160 \mathrm{~ms}$ considered in this study corresponds to nearly $35 \%$ of the stance period.

The four charts in Fig. 1 (right) reveal many interesting details on how the locomotion performance is sensitive to delayed detections and localization errors in the shin collision. We summarize them in the following observations:

Obs-1: The lower the friction between the shin and the obstacle, the more detrimental the shin collisions are to the locomotion performance. A lower contact friction leads to higher shin slippage accelerations that are difficult to stabilize even if the collision location is precisely known. This is seen in the top-left plot of Fig. 1 (right) in correspondence to zero systematic errors. Moreover, low contact frictions restrict the set of feasible stabilizing ground reaction forces and, in some cases, a feasible solution cannot be found to overcome the obstacle.

Obs-2: The higher the robot velocity, the less the shin collisions are detrimental to the robot motion. This relationship regards the impact of the shin collisions on the overall robot momentum, i.e., the higher the robot velocity, the higher the momentum and the less important the momentum disturbance caused by the shin collisions. This relationship can be noticed when observing the increased number of successful 5-trial sets between the top

bThe duty factor is the ratio between the stance duration and the duration of the gait cycle. 

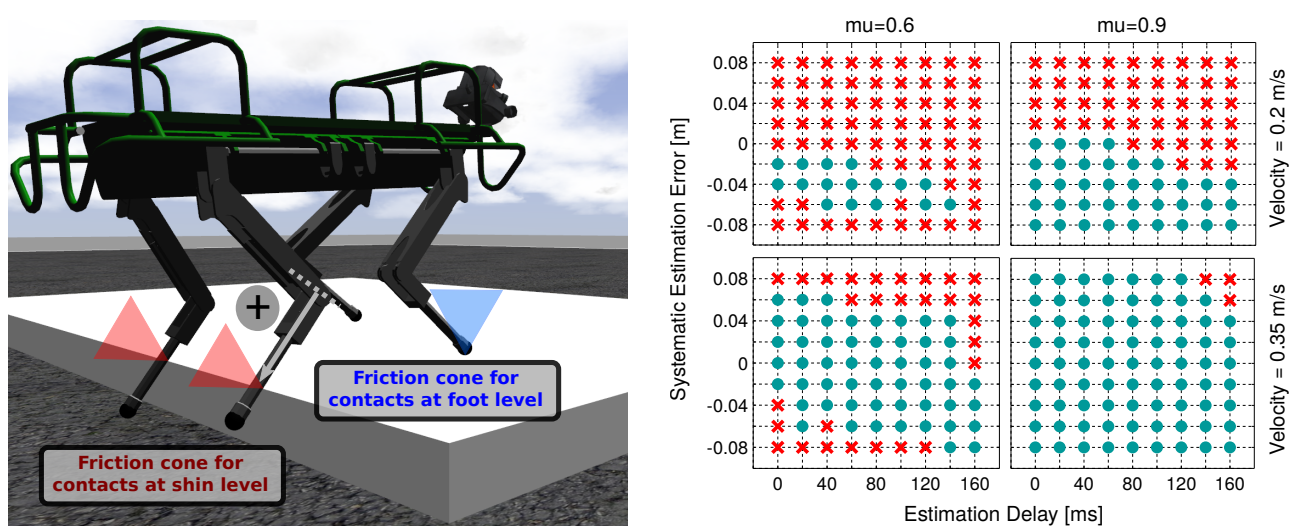

Fig. 1. Left) Simulation scenario used to study the effect of shin collision in quadruped locomotion during a walking trot. The translucent cones represent the friction cones where ground reaction forces should lie in order to avoid slippage. The gray arrow shows the sign convention for the shin contact location. Right) Effect of systematic errors and delays in the estimated shin contact position. Red cross markers and cyan dots show when the robot failed or succeeded, respectively, in climbing a $16 \mathrm{~cm}$ high platform. Top and bottom plots show the results associated to a robot forward velocity of $0.2 \mathrm{~m} / \mathrm{s}$ and $0.35 \mathrm{~m} / \mathrm{s}$, respectively. Left and right columns show the results associated to Coulomb friction coefficients of 0.6 and 0.9 , respectively.

and the bottom plots of Fig. 1 (right).

Obs-3: Positive and negative systematic errors have opposite effect on the locomotion performance. Positive errors are detrimental because they cause the robot to get stuck. This happens because: positive errors increase the chance of shin slippage (a larger shin lever arm makes the trunk controller generate larger joint torques and thus larger ground reaction forces); and positive errors create a momentum around the shin contact point that induces the knee to move (counter-clockwise in the figure) in a direction that is opposite to the robot motion. These effects are illustrated in Fig. 2 (a-b). However, negative systematic errors are likely to help the robot overcome the obstacle. This happens because they create a momentum around the shin contact point that tends to make the shin accommodate and slide on the surface without disturbing the motion. Since negative errors correspond to a lower shin contact lever arm, the trunk controller generates lower joint torques and thus lower ground reaction forces. See Fig. 2(c-d).

Obs-4: The higher the delay in the estimation of the contact, the higher the chance that a shin slippage occurs. This is because a delayed shin contact estimate, will always differ from the actual contact location with a positive estimation error. The resulting behavior is similar to the one described in Obs-3 for positive systematic errors with the difference that the side-effects are strongly dependent on the shin slippage velocity (i.e., for a given delay, the higher the slippage velocity, the higher the estimation error). This behavior is illustrated in Fig. 2(e-h).

Obs-5: Figure 1 (right) shows an interesting compensation effect that occurs between the delayed estimations and systematic errors. Looking at each of the plots, we can observe a trend of successful 5-trial sets along a diagonal strip from top-left to bottom-right corners of each plot (clearly seen in the bottom-left plot). We consider this behavior a compensation effect due to the fact that the negative systematic errors tend to compensate the positive estimation errors due to delayed estimates. This behavior is illustrated in Fig. 2(i-l) and represents an important finding of this study.

\section{A simple sensor-less approach for collision detection on distal limbs}

In this section, we propose a simple sensor-less approach based purely on the robot kinematics to detect when a single-point robot-environment interaction occurs at the distal limbs 

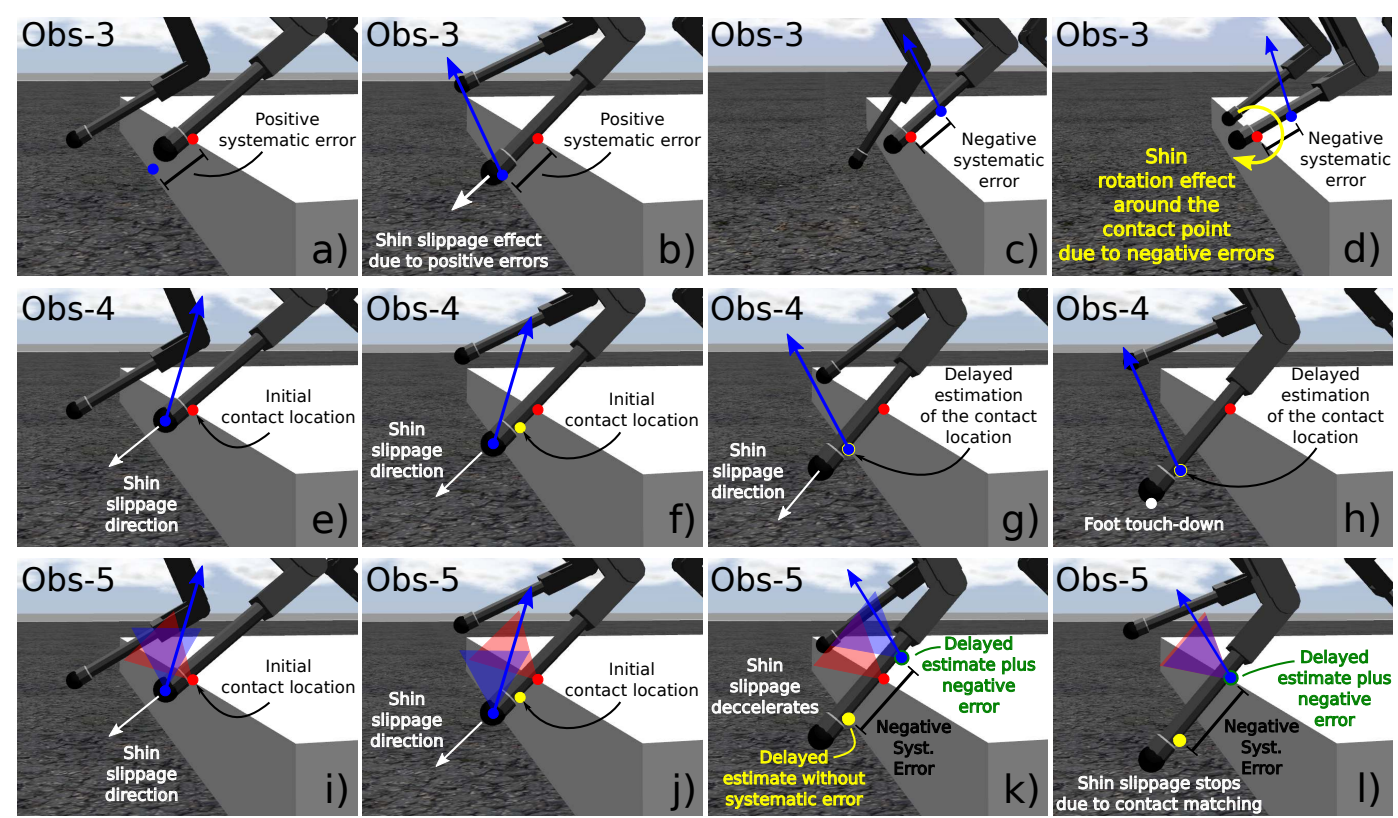

Fig. 2. Illustration of the behaviors described in the observations of Sec. 2: subfigures a) to b) and c) to d) illustrate the shin behavior when the contact location estimates present positive and negative systematic errors, respectively, and regard Obs-3; subfigures e) to h) are related to Obs-4 and illustrate the shin behavior when there is delay in the contact detection; subfigures i) to 1) regard Obs-5 and illustrate the compensation effect between the delayed detections and the presence of negative systematic errors.

(i.e., shins and forearms). Our approach relies on the assumption of a virtual element, a RRP kinematic structure (i.e., a spherical arm), where the prismatic joint represents the distal limb and the two rotary joints represent the contact point between the robot distal limb and the environment. As a proof of concept, we describe a reduced-order model that we applied to detect and localize a contact point at the distal links of quadruped robots, i.e., at the shins. The reduced model describes the virtual spherical arm in the robot's sagittal plane. The association of the states of the virtual arm and robot shin is illustrated in Fig. 3 for HyQ's left-hind leg (all virtual arm states are defined in the figure caption).

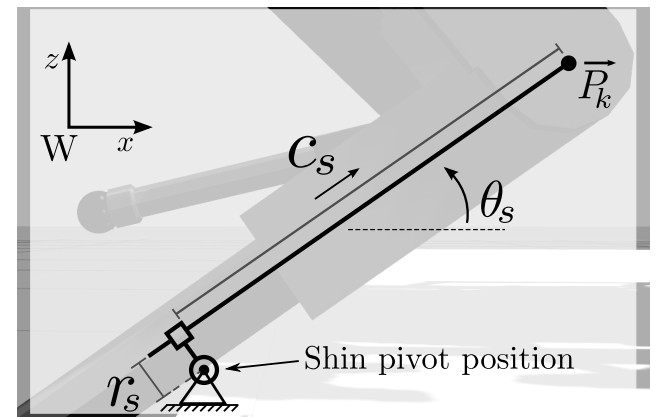

Fig. 3. Simplified model of the spherical arm by considering motions in the robot sagittal plane. The model is used to estimate the shin contact position $C_{s}$ and the shin slippage velocity $\dot{C}_{s}$ during a shin collision with an unknown obstacle. All variables are expressed in the world frame $\mathcal{W}$. The shin angle $\theta_{s}$ is defined as the angle between the horizontal plane (orthogonal to the gravity vector) and the robot shin in contact with the obstacle. The radius of the shin is $r_{s}$. The position of the knee with respect to the contact position is $P_{k}$.

According to the model illustrated in Fig. 3, the position of the knee $P_{k}=\left(P_{k_{x}}, P_{k_{z}}\right)$ 
with respect to the shin pivot position can be described as:

$$
\begin{aligned}
& P_{k_{x}}=r_{s} \cos \left(\pi / 2+\theta_{s}\right)+C_{s} \cos \left(\theta_{s}\right) \\
& P_{k_{z}}=r_{s} \sin \left(\pi / 2+\theta_{s}\right)+C_{s} \sin \left(\theta_{s}\right)
\end{aligned}
$$

Differentiating with respect to time and using trigonometric identities, the knee velocity can be computed as

$$
\begin{aligned}
& \dot{P}_{k_{x}}=-r_{s} \cos \left(\theta_{s}\right) \dot{\theta}_{s}+\dot{C}_{s} \cos \left(\theta_{s}\right)-C_{s} \sin \left(\theta_{s}\right) \dot{\theta}_{s} \\
& \dot{P}_{k_{z}}=-r_{s} \sin \left(\theta_{s}\right) \dot{\theta}_{s}+\dot{C}_{s} \sin \left(\theta_{s}\right)+C_{s} \cos \left(\theta_{s}\right) \dot{\theta}_{s}
\end{aligned}
$$

Since the goal is to find a description that connects the robot states with the states related to the collision, i.e., $C_{s}$ and $\dot{C}_{s}$, we rearrange (3) and (4) to obtain the following relationship

$$
\left[\begin{array}{c}
\dot{P}_{k_{x}}^{\prime} \\
\dot{P}_{k_{z}}^{\prime}
\end{array}\right]=\underbrace{\left[\begin{array}{cc}
\cos \left(\theta_{s}\right)-\sin \left(\theta_{s}\right) \dot{\theta}_{s} \\
\sin \left(\theta_{s}\right) & \cos \left(\theta_{s}\right) \dot{\theta}_{s}
\end{array}\right]}_{J_{s}}\left[\begin{array}{c}
\dot{C}_{s} \\
C_{s}
\end{array}\right]
$$

where $\dot{P}_{k_{x}}^{\prime}=\dot{P}_{k_{x}}+r_{s} \cos \left(\theta_{s}\right) \dot{\theta}_{s}, \dot{P}_{k_{z}}^{\prime}=\dot{P}_{k_{z}}+r_{s} \sin \left(\theta_{s}\right) \dot{\theta}_{s}$, and $J_{s}$ is the collision Jacobian. Given that estimates for $\dot{P}_{k}^{\prime}$ and $\theta_{s}$ can be provided by the robot state estimation, $C_{s}$ and $\dot{C}_{s}$ can be computed using the inverse of $J_{s}$, i.e.,

$$
\underbrace{\left[\begin{array}{c}
\dot{C}_{s} \\
C_{s}
\end{array}\right]}_{\hat{C}}=\underbrace{\left[\begin{array}{cc}
\cos \left(\theta_{s}\right) & \sin \left(\theta_{s}\right) \\
-\sin \left(\theta_{s}\right) \dot{\theta}_{s}^{-1} & \cos \left(\theta_{s}\right) \dot{\theta}_{s}^{-1}
\end{array}\right]}_{J_{s}^{-1}} \underbrace{\left[\begin{array}{c}
\dot{P}_{k_{x}}^{\prime} \\
\dot{P}_{k_{z}}^{\prime}
\end{array}\right]}_{\dot{P}_{k}^{\prime}}
$$

\section{Detecting and reacting to shin collisions using the proposed model}

To detect, localize and deal with shin collisions, we propose a simple strategy that uses both the estimated collision states $C_{s}$ and $\dot{C}_{s}$ and the observations described in Sec. 2. To detect the collision we consider the estimate $C_{s}$ and the slippage distance $\Delta_{s}$ defined as the integral of $\dot{C}_{s}$ with respect to the time elapsed from a detected leg stance phase (e.g., in HyQ the stance phase is detected from joint torque sensors).

Given $C_{s}$ and $\Delta_{s}$, a detection is triggered when $\Delta_{s}$ is higher than a specific threshold or when $C_{s}$ is consistent with the shin length. Due to the fact that $C_{s}$ tends to be a noisy estimate, we consider it only when its covariance enters an acceptable range and the correspondent shin angular rate and knee velocity estimates present a magnitude that leads to an acceptable signal-to-noise ratio.

When the collision is detected through $C_{s}$, the localization of the contact at the shin is automatically acquired. However, such information is not known when the detection is triggered through $\Delta_{s}$. In this case, using the outcomes from Obs-3 and Obs-4, we assume an initial contact location $C_{s 0}$ that is closer to the knee than the foot. This increases the chances for the localization error to be negative. Obs-4 is linked to this process because $\Delta_{s}$ is the result of an integration, and thus has inherent delay.

As a reaction, the estimated shin contact location and the shin orientation are fed back into the stabilizing algorithm (trunk controller) to properly distribute the ground reaction forces. In the next section we show experimental results of the application of this approach.

\section{Experimental results}

We implemented the strategy proposed in Sec. 4 on the HyQ robot. The proposed strategy is implemented inside the Reactive Controller Framework ${ }^{14}$ that generates and controls the robot motion for a blind walking trot gait. In this experiment, a step is placed in between 
the robot front and hind legs and the robot is commanded to trot backwards at $0.35 \mathrm{~m} / \mathrm{s}$ without the knowledge of the environment. Figure 4 shows the snapshots of the experiments to compare locomotion performance with and without the detection and contact feedback from the proposed strategy.

For the detection criteria based on $C_{s}$, we consider a minimum $C_{s}$ covariance of $0.005 \mathrm{~m}$, minimum shin angular rate of $0.05 \mathrm{rad} / \mathrm{s}$, and a minimum shin tangential velocity of $0.15 \mathrm{~m} / \mathrm{s}$. For the detection based on $\Delta_{s}$, we define a minimum slippage distance of $0.02 \mathrm{~m}$ and an initial contact estimate $C_{s 0}=0.15 \mathrm{~m}$. All these parameters are chosen based on the accuracy and noise of the signals provided by the robot state estimator. The more accurate the state estimation outputs, the lower the magnitude of the thresholds.

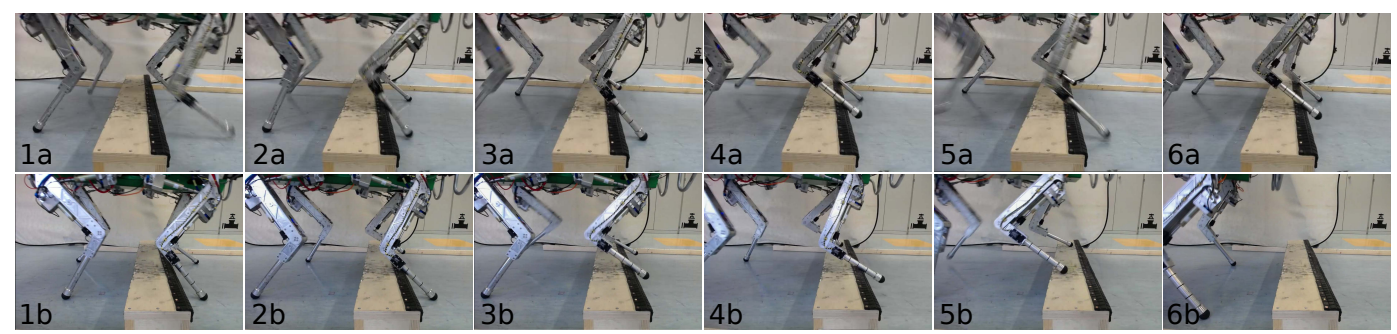

Fig. 4. Snapshots of the experimental tests where $\mathrm{HyQ}$ is performing a blind walking trot backwards. a) The top snapshots show the performance when there is no feedback about the shin contacts. In this case, HyQ gets stuck because both front shins cannot stop sliding on the step (see 3a-6a). b) The bottom snapshots show the performance of the proposed approach where contact information is detected and fed back to the trunk controller. The robot successfully overcomes the step by stopping the shin slippage during the collisions (see $2 \mathrm{~b}$ and $3 \mathrm{~b}$ ). Link to the video of the experimental tests: https://youtu.be/vHc5ZpkCy8Y

The bottom snapshots in Fig. 4 show the motion sequence of a successful test where two shin collisions are detected and fed back into the trunk controller. These two collisions happen at snapshots $2 \mathrm{~b}$ and $3 \mathrm{~b}$. Figure 5 shows the most relevant detection and estimated variables during such collisions. We note that by observing the slippage distance $\Delta_{s}$ the shin slippage stops after the collision is detected. This allows the robot to properly use the shin contact, and overcome the step, by better distributing the ground reaction forces.

The robustness and performance of the proposed approach are not directly dependent on the gait the robot executes, but rather on the accuracy of the robot state estimates (i.e., the estimates of the linear and angular velocities of the robot trunk). These estimates are used to obtain $\dot{P}_{k}^{\prime}, \theta_{s}$ and $\dot{\theta}_{s}$, which directly affect the estimate of $C_{s}$, as described in (6). The estimate of $\dot{C}_{s}$ is more stable, since it does not depend on $\dot{\theta}_{s}$, and it is likely to be the one detecting the shin contact, through $\Delta_{s}$, when the accuracy of the robot state estimator is degraded.

\section{Conclusions}

In this paper, we presented a pioneer study on how delays and systematic errors, which are present in shin contact detection and localization algorithms, affect the performance of a dynamic quadruped gait. Detailed observations were described and illustrated. One of the major outcomes of these observations is that negative systematic errors are much less detrimental and, in some cases, can be intentionally included to compensate for delayed detections. Furthermore, we proposed a generic kinematics-based model to estimate contact location at robot distal limbs, and described a reduced model to localize contact points on quadruped shins. The proposed model and the outcomes of the study were put together to build a simple and effective strategy that was tested on a real robot to overcome a step during blind locomotion. 


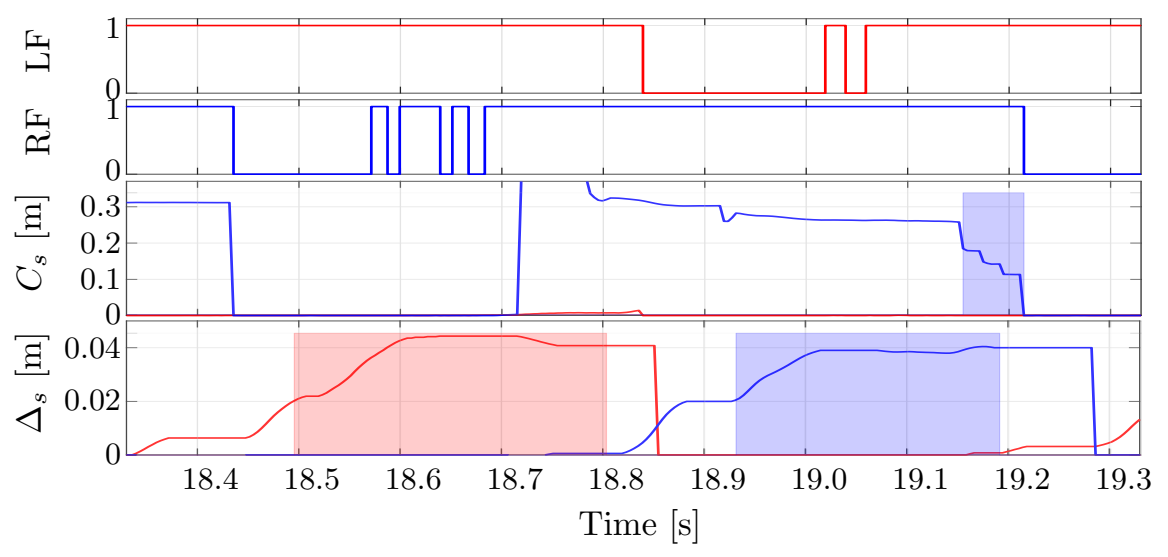

Fig. 5. Detection and estimation signals for the experimental test shown in Fig. 4, with the time scale that reports the two collisions that happen during the snapshots $2 \mathrm{~b}$ and $3 \mathrm{~b}$ : the snapshot $2 \mathrm{~b}$ shows a shin collision for the left-front (LF) leg and the snapshot $3 b$ shows a shin collision for the right-front (RF) leg. The two top plots shows the stance status detected for both LF and RF legs. The translucent areas show when the collision is detected through the $C_{s}$ estimate (i.e., $C_{s}$ estimate satisfying the criteria described in Sec. 4) and through $\Delta_{s}$ (i.e., when the slippage distance is higher than its threshold). Red and blue colors represent the $\mathrm{LF}$ and $\mathrm{RF}$ associated signals, respectively.

\section{References}

1. T. Koolen, S. Bertrand, G. Thomas, T. de Boer, T. Wu, J. Smith, J. Englsberger and J. Pratt, Design of a Momentum-Based Control Framework and Application to the Humanoid Robot Atlas International Journal of Humanoid Robotics 132016.

2. S. Fahmi, C. Mastalli, M. Focchi and C. Semini, Passive Whole-body Control for Quadruped Robots: Experimental Validation over Challenging Terrain IEEE RAL 2019.

3. C. D. Bellicoso, F. Jenelten, C. Gehring and M. Hutter, Dynamic Locomotion through Online Nonlinear Motion Optimization for Quadrupedal Robots IEEE RAL 37662018.

4. A. D. Prete, F. Nori, G. Metta and L. Natale, Control of contact forces: The role of tactile feedback for contact localization, in IEEE/RSJ International Conference on Intelligent Robots and Systems, 2012.

5. S. Ivaldi, M. Fumagalli, M. Randazzo, F. Nori, G. Metta and G. Sandini, Computing robot internal/external wrenches by means of inertial, tactile and $\mathrm{F} / \mathrm{T}$ sensors: Theory and implementation on the iCub IEEE/RAS International Conference on Humanoid Robots (IEEE, 2011).

6. Y. Ohmura, Y. Kuniyoshi and A. Nagakubo, Conformable and scalable tactile sensor skin for curved surfaces, in IEEE International Conference on Robotics and Automation, 2006.

7. J. Ulmen and M. Cutkosky, A robust, low-cost and low-noise artificial skin for human-friendly robots, in IEEE International Conference on Robotics and Automation, 2010.

8. A. de Luca and R. Mattone, Sensorless Robot Collision Detection and Hybrid Force/Motion Control, in IEEE Int. Conf. on Robotics and Automation, apr 2005.

9. A. De Luca, A. Albu-Schäffer, S. Haddadin and G. Hirzinger, Collision detection and safe reaction with the DLR-III lightweight manipulator arm IEEE International Conference on Intelligent Robots and Systems 2006.

10. J. Vorndamme, M. Schappler and S. Haddadin, Collision detection, isolation and identification for humanoids IEEE International Conference on Robotics and Automation 2017.

11. L. Manuelli and R. Tedrake, Localizing external contact using proprioceptive sensors: The Contact Particle Filter, in IEEE/RSJ Int. Conf. on Intelligent Robots and Systems, 2016.

12. M. Focchi, R. Orsolino, M. Camurri, V. Barasuol, C. Mastalli, D. G. Caldwell, and C. Semini, Heuristic Planning for Rough Terrain Locomotion in Presence of External Disturbances and Variable Perception Quality Springer Track in Advanced Robotics series 2019.

13. C. Semini, N. G. Tsagarakis, E. Guglielmino, M. Focchi, F. Cannella and D. G. Caldwell, Design of HyQ - a hydraulically and electrically actuated quadruped robot Proceedings of the Institution of Mechanical Engineers, Part I: Journal of Syst. and Control Eng. 2252011.

14. V. Barasuol, J. Buchli, C. Semini, M. Frigerio, E. R. De Pieri and D. G. Caldwell, A reactive controller framework for quadrupedal locomotion on challenging terrain IEEE International Conference on Robotics and Automation 2013. 